\title{
Betting the Farm
}

The approach to clinical conundrums by an expert clinician is revealed through the presentation of an actual patient's case in an approach typical of a morning report. Similarly to patient care, sequential pieces of information are provided to the clinician, who is unfamiliar with the case. The focus is on the thought processes of both the clinical team caring for the patient and the discussant.

This icon represents the patient's case. Each paragraph that follows represents the discussant's thoughts.

\author{
Ayokunle Abegunde, $\mathrm{MD}^{1}$, Emad Hakemi, $\mathrm{MD}^{2}$, Benjamin Mba, MD² , Andrew Odden, $\mathrm{MD}^{3}$, Robert A. Weinstein, MD²
}

${ }^{1}$ Department of Medicine, Oklahoma University Health Sciences Center, Oklahoma City, Oklahoma; ${ }^{2}$ Department of Medicine, John H. Stroger, Jr. Hospital of Cook County, Chicago, Illinois; ${ }^{3}$ Department of Medicine, Washington University School of Medicine, St. Louis, Missouri.

A 65 -year-old man with a 6-month history of diabetes mellitus presented to the emergency department in May with 1 week of fevers, headaches, myalgia, polydipsia, and polyuria.

The patient presents with symptoms suggestive of uncontrolled diabetes and infection. The broad diagnostic categories include acute infection, an emerging chronic process aggravating his diabetes, or a noninfectious mimic such as autoimmune disease or lymphoproliferative disease. New onset headache in an older patient is concerning. Although it may be attributed to fever and dehydration, primary central nervous system processes such as meningitis or encephalitis must be considered. At this stage, a detailed exposure history, including travel, food, pets, hobbies, and sick contacts as well as occupation and national origins is needed. This patient presented in May, making illnesses that peak in other seasons such as influenza and West Nile fever less likely.

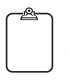

He had no other medical problems except diabetes. He was not taking any medications; he had been started on glipizide but had stopped taking it 1 month prior. He denied fever, cough, chest pain, palpitations, abdominal pain, nausea, vomiting, dysuria, focal weakness, visual changes, or photophobia. He was born in Mexico and emigrated at the age of 25 years. Two months prior to presentation he visited a cattle farm in Mexico; he denied any direct contact with farm animals or dairy products. He denied ill contacts, pets, known tuberculosis exposures, and sexual partners other than his wife.

The history of recent travel to Mexico with a visit to a farm raises concerns about zoonoses. The endemic zoonoses that should be considered include parasitic (toxoplasmosis), fungal (coccidiodomycosis), and bacterial (brucellosis, Q fever, leptospirosis, tularemia, salmonellosis) infections. Nonzoonotic granulomatous infections such as cytomegalovirus (CMV) and Epstein-Barr virus (EBV), mycobacteria, fungi

*Address for correspondence and reprint requests: Benjamin Mba, MD, Department of Medicine, John H. Stroger, Jr. Hospital of Cook County, 1900 W Polk Street, Suite 1518, Chicago, IL 60612; Telephone: 312-864-7231; Fax: 312-864-9725; E-mail: bmba@cookcountyhhs.org

Additional Supporting Information may be found in the online version of this article.

Received: July 11, 2015; Revised: December 18, 2015; Accepted: December 22, 2015

2016 Society of Hospital Medicine DOI 10.1002/jhm.2548

Published online in Wiley Online Library (Wileyonlinelibrary.com). (histoplasmosis, blastomycosis, cryptococcosis, aspergillosis), and bacteria (actinomycosis) should also be considered.

On examination, he was an elderly Hispanic male who appeared ill but in no acute distress. He was overweight, with a BMI of 29 . His temperature was $39^{\circ} \mathrm{C}$, pulse 66 beats/ minute, blood pressure 108/68 $\mathrm{mm} \mathrm{Hg}$, respiratory rate 18 per minute, and oxygen saturation was $96 \%$ on room air. There were no ulcerations, exudates, or erythema in the oropharynx. There was no sinus tenderness or lymphadenopathy. Cardiac examination revealed normal heart sounds with no murmurs. Respiratory examination demonstrated clear lungs. His abdomen was soft and nontender, whereas the liver and spleen were not palpable. There was no nuchal rigidity, and his mental status was normal. There were no cranial nerve deficits or weakness in his extremities. There was no skin rash or peripheral stigmata of infectious endocarditis. Genitourinary examination revealed no ulcerations, inguinal lymphadenopathy, or urethral discharge. There was no tenderness, warmth, or erythema on examination of all joints.

The physical exam is notable for temperature-pulse dissociation. Heart rate should increase by about 10 beats/minute for every 1-degree increase in Fahrenheit temperature. The infectious causes of temperature-pulse dissociation are largely intracellular pathogens such as Salmonella, Coxiella, Chlamydia, Leptospira, Legionella, Francisella, Mycoplasma, and dengue virus. This patient is at increased risk for infection by any of these pathogens based on his recent travel to Mexico. Drug fever is the most common noninfectious cause of temperature-pulse dissociation, but this patient took no medications. At this point, a complete blood count and differential, urinalysis, blood cultures, chest $\mathrm{x}-$ ray, and electrocardiogram should be ordered. Testing for human immunodeficiency virus (HIV) is appropriate, as up to $50 \%$ of patients with newly diagnosed HIV have no acknowledged risk factors. Serological studies for the aforementioned pathogens may be indicated depending on the results of these initial diagnostic tests.

Serum sodium concentration was $122 \mathrm{mEq} / \mathrm{L}$, potassium $4.0 \mathrm{mEq} / \mathrm{L}$, chloride $88 \mathrm{mEq} / \mathrm{L}$, bicarbonate 14 $\mathrm{mEq} / \mathrm{L}$, blood urea nitrogen $17 \mathrm{mg} / \mathrm{dL}$, creatinine $0.7 \mathrm{mg}$ / $\mathrm{dL}$, glucose $402 \mathrm{mg} / \mathrm{dL}$, and calcium $8.5 \mathrm{mg} / \mathrm{dL}$. Total protein was $5.4 \mathrm{~g} / \mathrm{dL}$, albumin $2.9 \mathrm{~g} / \mathrm{dL}$, total bilirubin 0.9 $\mathrm{mg} / \mathrm{dL}$, direct bilirubin $0.4 \mathrm{mg} / \mathrm{dL}$, alkaline phosphatase 126 U/L (normal 53-128), gamma-glutamyl transferase $264 \mathrm{U} / \mathrm{L}$ (normal 3-60), aspartate aminotransferase $51 \mathrm{U} / \mathrm{L}$ (normal 8-40), alanine aminotransferase $62 \mathrm{U} / \mathrm{L}$ (normal 5-56), and lactate dehydrogenase $248 \mathrm{U} / \mathrm{L}$ (normal 85-210). The white 
blood cell (WBC) count was $6800 \mathrm{~mm}^{3}$ (51\% band forms, $38 \%$ segmented neutrophils, $6 \%$ monocytes, $5 \%$ lymphocytes). The hemoglobin was $15.7 \mathrm{~g} / \mathrm{dL}$, with mean corpuscular volume (MCV) of $102 \mathrm{fL}$ and platelet count 59,000/ $\mathrm{mm}^{3}$. Peripheral-blood smear showed occasional macrocytes. Prothrombin time was $\mathbf{1 3 . 6}$ seconds and partial thromboplastin time was 34.5 seconds. C-reactive protein was $11.8 \mathrm{mg} / \mathrm{dL}$. Urinalysis revealed $80 \mathrm{mg}$ of ketones per deciliter, no cells, and nitrite was negative. Hemoglobin A1c was $13 \%$, and HIV antibody testing was negative.

Elevated circulating bands and thrombocytopenia suggest infection; however, bone marrow infiltration by infectious or neoplastic process is also possible and should be investigated. The increased gamma-glutamyl transferase, alkaline phosphatase, and mild increases in transaminases suggest hepatic pathology. The combination of unexplained fever, hyponatremia, thrombocytopenia, elevated liver enzymes, and travel to Mexico mandates investigation for infectious diseases that often involve both the bone marrow and liver such as Brucella, Coxiella, and fungal infections such as histoplasmosis. Autoimmune diseases such as systemic lupus erythematosus and malignancy should also be considered. Blood cultures should be incubated beyond the usual 5 days because of the slower growth of Brucella or Salmonella typhi. An HIV viral load should be obtained to evaluate for acute retroviral syndrome. Serologic tests for Rickettsia, Coccidiodes, and hepatitis $\mathrm{A}, \mathrm{B}$, and $\mathrm{C}$ viruses should be obtained. Urine should be tested for Histoplasma and Legionella antigens. Abdominal imaging should be obtained to evaluate for hepatobiliary disease, occult intra-abdominal abscess, or malignancy. Because the patient has unexplained fever and headache, imaging of the central nervous system and lumbar puncture are warranted.

His diabetic ketoacidosis (DKA) was treated with intravenous fluids and insulin. Lumbar puncture and cerebrospinal fluid (CSF) analysis revealed opening pressure of $18 \mathrm{~cm} \mathrm{H}_{2} \mathrm{O}$ (normal 10-25), cell count WBC 3/ $\mu \mathrm{L}$ (normal $0-5$ ), red blood cell 204/ $\mu \mathrm{L}$ (normal 0), CSF protein $25 \mathrm{mg}$ / dL (normal 20-50), and glucose $68 \mathrm{mg} / \mathrm{dL}$ (normal 50-70). Blood cultures showed no growth. HIV RNA was undetectable. Hepatitis $\mathrm{C}$ antibody was negative, and hepatitis $\mathrm{A}$ and $B$ serologies were not consistent with an acute infection. Serum ferritin was $1147 \mathrm{ng} / \mathrm{mL}$. Histoplasma and Legionella urine antigen tests were negative. CMV, EBV, and herpes simplex virus DNA were not detected in blood samples. Anti-neutrophil antibody, anti-mitochondrial antibody and anti-neutrophil cytoplasmic antibodies were undetectable. Anti-smooth muscle antibody was positive at a titer of 1:80. Transthoracic echocardiogram revealed normal heart valves without vegetations. A chest radiograph was normal. Brain computed tomography (CT) revealed atrophic frontal lobes. CT of his chest, abdomen, and pelvis demonstrated focal inflammatory changes of a loop of distal small bowel with surrounding fluid collection, suggesting small bowel diverticulitis. There were no pulmonary infiltrates noted, and the remainder of the CT was unremarkable.

Because the patient remains ill and additional serological test results will take time to return, a key consideration at this point is empiric treatment while awaiting test results. The CSF examination was normal. A history of travel including animal and tick exposures should be reevaluated.
The timing of the trip to Mexico was outside the usual incubation period for many pathogens except for Coxiella or Brucella, and empiric therapy for both would be appropriate. The abdominal CT suggests small bowel diverticulitis, which is a rare clinical entity.

The benign abdominal examination suggests the finding is incidental. However, there are several infections that may involve the distal small bowel and proximal colon, such as yersiniosis, salmonellosis, tuberculosis, actinomycosis, histoplasmosis, and noninfectious processes including Crohn's disease and neoplasia. The absence of diarrhea or hematochezia makes yersiniosis, salmonellosis, and Crohn's disease unlikely. Histoplasmosis is unlikely given the negative urine antigen. Evaluation for neoplasia of the distal small bowel requires histologic examination. A colonoscopy with random biopsies of the colon and terminal ileum is the next step if other tests are unrevealing.

The patient was empirically treated for small bowel diverticulitis with ceftriaxone and metronidazole. Because of continued daily fevers as high as $39^{\circ} \mathrm{C}$, his therapy was changed to vancomycin and piperacillintazobactam to cover methicillin-resistant Staphylococcus aureus and resistant gram-negative bacilli. The patient developed new scleral icterus on hospital day 6; the remainder of his examination was unchanged. Serum sodium concentration was $127 \mathrm{mEq} / \mathrm{L}$, potassium $2.7 \mathrm{mEq} / \mathrm{L}$, phosphorus $1.3 \mathrm{mg} / \mathrm{dL}$, magnesium $1.6 \mathrm{mg} / \mathrm{dL}$, total bilirubin $5.6 \mathrm{mg} / \mathrm{dL}$, direct bilirubin $3.6 \mathrm{mg} / \mathrm{dL}$, alkaline phosphatase $193 \mathrm{U} / \mathrm{L}$, gamma-glutamyl transferase $300 \mathrm{U} / \mathrm{L}$, aspartate aminotransferase $91 \mathrm{U} / \mathrm{L}$, alanine aminotransferase $52 \mathrm{U} / \mathrm{L}$. Brucella serology was negative.

His liver enzymes remain elevated with new onset jaundice consistent with hepatitis and intrahepatic cholestasis. His persistent hypophosphatemia, hypokalemia, and hypomagnesaemia well after resolution of diabetic ketoacidosis suggests acute tubulointerstitial dysfunction, which may be a complication of empiric antibiotic treatment or renal involvement by his underlying condition. Additional blood cultures, and tissue examination and culture are the next appropriate steps. Liver or bone marrow biopsy may suggest a diagnosis that can be confirmed by tissue culture or immunohistochemistry. Histologic findings such as fibrin ringed granulomas, caseating or noncaseating granulomas, or lymphomatous infiltration may suggest Coxiella ( $Q$ fever), tuberculosis, or lymphoma respectively. Because a liver biopsy is invasive and usually provides less tissue for culture, bone marrow examination should be obtained first.

A gallium 67 scan showed nonhomogenous increased uptake in both lungs and kidneys, consistent with interstitial nephritis and bilateral pneumonia. Serum protein electrophoresis demonstrated a monoclonal immunoglobulin (Ig)G lambda band with a kappa/lambda ratio of 0.9 (normal 1.4-2.8). Bone marrow biopsy showed normal hematopoiesis; no plasma or malignant cells, granulomas, or evidence of hemophagocytosis; and fungal and mycobacterial stains and cultures were negative. Colonoscopy revealed normal-appearing mucosa. Histologic examination and culture of random biopsies from the colon and terminal ileum were negative for fungi, viruses, and mycobacteria. An ultrasound-guided liver biopsy revealed numerous noncaseating granulomas formed of histiocytes and neutrophils 
with occasional fibrin rings. Fungal, viral, and mycobacterial stains and cultures were negative. The patient's fever resolved after 14 days, and he was discharged home without a diagnosis and close outpatient follow-up.

The hepatic granulomas with fibrin rings are highly suggestive of $\mathrm{Q}$ fever, although ring granulomas may be seen in tuberculosis, typhoid fever, lymphoma, drug reactions, sarcoidosis, and CMV infections. Competing diagnoses such as CMV have been excluded by negative serology. Microscopic examination, tissue staining, and culture from liver and bone marrow biopsies were negative for $S$ typhi, mycobacteria, and lymphoma. Gallium scan findings are generally nonspecific and of little utility in cases such as this. The kidney involvement correlates with the biochemical evidence of tubulointerstitial dysfunction; pulmonary involvement may reflect subclinical pulmonary infection with Coxiella. Given the normal bone marrow biopsy, the monoclonal gammopathy is of undetermined significance. The positive antismooth muscle antibody can be related to $\mathrm{Q}$ fever. Antismooth muscle antibodies frequently occur in Q fever, especially in those patients with hepatitis. Given the history of exposure to cattle, unexplained fever with temperature-pulse dissociation and liver biopsy findings, Q fever is the most likely diagnosis and empiric treatment with doxycycline is warranted.

Results of serology for Coxiella burnetii sent during admission were returned after the patient's discharge. C burnetii phase I IgG and IgM antibody titers were positive (1:512 each). C burnetii phase II IgG and IgM titers also were positive (1:1024 each). The patient was seen within a week and started on doxycycline $100 \mathrm{mg}$ twice daily for 2 weeks for acute $\mathrm{Q}$ fever. His symptoms improved; hyponatremia, liver function tests, and thrombocytopenia normalized after treatment.

\section{DISCUSSION}

Q fever was first described in 1937 as a febrile illness affecting Australian slaughterhouse workers. ${ }^{1}$ The "Q" in $\mathrm{Q}$ fever stands for "query" and reflected the initial uncertainty surrounding the underlying cause of the illness. The causative organism, C burnetti, is an obligate intracellular bacterium that resides within macrophage lysosomes. It can be found in the urine, feces, milk, placenta, and amniotic fluid of ungulates (cattle, sheep, and other ruminants), and other animals such as domestic cats and dogs. C burnetii is transmitted via inhalation, ingestion, occupational, or common source exposures, and in 1 case report by person-to-person sexual transmission. ${ }^{2}$ In addition to slaughterhouse workers, pregnant women and immunosuppressed patients are more susceptible to developing $\mathrm{Q}$ fever. ${ }^{3}$ For patients with suspected $\mathrm{Q}$ fever, a detailed occupational history, including specific job duties and potential exposure to animal products, is imperative.

Q fever has both acute and chronic presentations, which are differentiated based on the clinical illness and serologies. The symptoms of acute $\mathrm{Q}$ fever are nonspecific and may include influenza-like illness, fever, pneumonia, and hepatitis. It presents less commonly with hemolytic anemia, interstitial nephritis, monoclonal gammopathy, or aseptic meningitis. ${ }^{4-7}$ Symptoms typically begin between 1 and 3 weeks after animal exposure and may persist for several months. Chronic Q fever occurs when unrecognized or untreated infection persists for greater than 6 months. It commonly presents with culture-negative endocarditis, although infected aneurysms, osteomyelitis, or other distant sites of infection may also occur.

C burnetti is present in 2 antigenic forms that can be assessed by serology. Phase I is the more virulent, infectious form of $C$ burnetti, which transitions to the avirulent phase II form during laboratory handling. In acute Q fever, phase II serologies are typically elevated out of proportion to phase I serologies, whereas this pattern is reversed in chronic Q fever. The diagnostic gold standard of acute Q fever is a 4-fold rise in phase II antibody titers taken 3 to 6 weeks apart. ${ }^{8}$ Histologic examination of affected organs can support a diagnosis of $\mathrm{Q}$ fever. The presence of ringed granulomas on liver or bone marrow biopsy specimens is highly suggestive, but not pathognomonic, of $\mathrm{Q}$ fever. ${ }^{9}$

$\mathrm{Q}$ fever is highly susceptible to several classes of antibiotics. For acute Q fever, doxycycline and tetracycline are typically used, with fluoroquinolones and chloramphenicol as alternatives. ${ }^{8,10}$ Patients with chronic Q fever should be treated with doxycycline and hydroxychloroquine. The addition of hydroxychloroquine alkalinizes the macrophage lysosome and enhances bacterial eradication. ${ }^{8}$ For patients with acute $\mathrm{Q}$ fever, physicians should determine the risk of progression to chronic Q fever because closer monitoring is necessary. Patients with valvular heart lesions, immunosuppression, and pregnant women are at elevated risk of chronic Q fever. Trimethoprim/sulfamethoxazole can be used in place of doxycycline in pregnant women, as doxycycline and fluoroquinolones are contraindicated in pregnancy. ${ }^{8}$

This patient presented with a nonspecific febrile illness. Although the treating clinicians obtained a history of exposure to cattle early in his course, both the diagnosis and treatment were delayed. There are several possible explanations for the delay. First, although Q fever is a relatively common zoonosis, it remains an uncommon diagnosis, particularly among hospitalized patients. As a result, clinicians often focus on more common conditions. In this case, typical infections, malignancies, and inflammatory diseases were considered more likely. Second, the patient presented with hepatitis, an uncommon presentation of Q fever. Classical clinical reasoning suggests that atypical presentations of common diseases will occur more frequently than typical presentations of uncommon diseases. This case presented with an atypical presentation of an uncommon disease. The resultant lower pretest probability further dissuaded the patient's physicians from consideration of $\mathrm{Q}$ fever. Third, the finding of small bowel diverticulitis was a potential distractor. In patients with nonspecific febrile illnesses, it is common for physicians to anchor on any abnormal findings. In this case, the small bowel diverticulitis led to antibiotic treatment that was ineffective against $C$ burnetti.

There were several clues to the diagnosis of $\mathrm{Q}$ fever in this patient's presentation. First, the pulse-temperature dissociation suggested infection with an intracellular pathogen. Hospitalists should recognize this association and be mindful of this often-subtle clinical finding when faced with diagnostic uncertainty. Second, the patient was exposed to cattle prior to the onset of his illness. The fact that he did not have a direct exposure to animals underscores the infectivity of $C$ burnetti. Finally, elevated alkaline phosphatase and transaminases were suggestive of an infiltrative disease; in the 
setting of a nonspecific febrile illness, Q fever was an important diagnostic consideration.

The key treatment decision in this case was the initiation and choice of antibiotics. Because of this patient's history of exposure to cattle and lack of a compelling alternative diagnosis, empiric treatment with doxycycline would have been appropriate. Hospitalists must weigh the potential benefit of early treatment of $\mathrm{Q}$ fever against the risks associated with antibiotic overuse. In patients presenting with a febrile illness after ungulate exposure, the decision to "bet the farm" with empiric doxycycline therapy may lead to clinical improvement, obviating a more invasive or extensive diagnostic evaluation.

\section{TEACHING POINTS}

1. Acute $Q$ fever typically presents 2 to 3 weeks after ungulate exposure with a febrile illness, pneumonia, and granulomatous hepatitis.

2. Pulse-temperature dissociation is suggestive of infection by intracellular pathogens such as Coxiella, Salmonella, Leptospira, Legionella, and Mycoplasma.

3. Clinicians should consider empiric doxycycline therapy in patients with suspected zoonosis (eg, Q fever, brucellosis, anaplasmosis, leptospirosis, Rocky Mountain spotted fever) while awaiting confirmatory tests, as improvement may obviate invasive testing.

Disclosure: Nothing to report.

\section{References}

1. Derrick EH. "Q" fever, a new fever entity: clinical features, diagnosis, and laboratory investigation. Rev Infect Dis. 1983;5(4):790-800.

2. Madariaga MG, Rezai K, Trenholme GM, Weinstein R. Q fever: a biological weapon in your backyard. Lancet Infect Dis. 2003;3(11): 709-721.

3. Tissot-Dupont H, Vaillant V, Rey S, Raoult D. Role of sex, age, previous valve lesion, and pregnancy in the clinical expression and outcome of Q fever after a large outbreak. Clin Infect Dis. 2007;15:44(2):232237.

4. Korkmaz S, Elaldi N, Kayatas M, Sencan M, Yildiz E. Unusual manifestations of acute $\mathrm{Q}$ fever: autoimmune hemolytic anemia and tubulointerstitial nephritis. Ann of Clin Microbiol Antimicrob. 2012;11:14.

5. Parker N, Barralet J, Bell A. Q fever. Lancet. 2006;367(9511):679688.

6. Montejo M, Aguirrebengoa K, Ruiz-Irastorza G, Urkijo JC, de la Prieta R, Aguirre C. Transitory monoclonal gammopathy and acute Q fever. Enferm Infecc Microbiol Clin. 1995;13(7):442.

7. Maurin M, Raoult D. Q fever. Clin Microbiol Rev. 1999;12(4):518-553.

8. Anderson A, Bijilmer H, Fournier PE, et al. Diagnosis and management of Q fever-United States, 2013: recommendations from CDC and the Q Fever Working Group. MMWR Recomm Rep. 2013; 62(RR-03):1-30.

9. Marazuela M, Moreno A, Yebra M, et al. Hepatic fibrin-ring granulomas: a clinicopathologic study of 23 patients. Hum Pathol. 1991; 22(6):607-613.

10. Leshem E, Meltzer E, Schwartz E. Travel-associated zoonotic bacterial diseases. Curr Opin Infect Dis. 2011;24(5):457-463. 